\title{
Etude de critères de sélection pour une résis- tance générale à la verticilliose chez la tomate
}

\author{
Issa BÉYE \& Jean-François LAFAY \\ Laboratoire de Cryptogamie associé au C.N.R.S. (LA. 115), Université de Paris-Sud, Bâtiment 400 \\ F 91405 Orsay Cedex
}

Mots clés additionnels : Résistance générale, composantes, notations, croissance, floraison, symptômes foliaires.

Additional key words : General resistance, components, notation, growth, flowering, foliar symptoms.

\section{INTRODUCTION}

L'amélioration de la résistance des plantes cultivées aux maladies d'origine parasitaire tire un large parti $\mathrm{du}$ polymorphisme des populations naturelles des espèces sauvages auxquelles elles sont apparentées.

Ainsi, Lycopersicon pimpinellifolium (Jusl.) Mill. fournit à la tomate des gènes de résistance à des affections fongiques (Fusarium oxysporum f. sp. lycopersici (Sacc.) Snyder \& Hansen, Cladosporium fulvum Cke., Verticillium dahliae Kleb. et $V$. albo-atrum Reinke \& Berth., Phytophthora infestans (Mont.) de
Bary), bactériennes (Corynebacterium michiganense (E. F. Sm.) Jens., Pseudomonas tomato E. F. Sm.) et virales (spotted wilt, tobacco yellow leaf curl). D'autres sources de résistance sont disponibles chez d'autres espèces comme Lycopersicon hirsutum Humb \& Bonpl. (C. fulvum, virus de la mosaïque du tabac, C. michiganense) et L. peruvianum (L.) Mill. (C. fulvum, V.M.T., Meloüdogyne incognita (Kufoid \& White) Chitwood). Certaines de ces résistances ne sont pas complètes. Par exemple, le niveau d'expression du gène $\mathrm{Ph} 2$ de résistance de la tomate à $P$. infestans dépend de la luminosité (ClerJEAu et al., 1979). 
La détection des formes spécifiques de résistance dans les populations naturelles et leur introduction chez des variétés d'intérêt agronomique posent assez peu de problèmes : les critères sont qualitatifs, la plante présente ou non, selon le génotype de l'agent pathogène, une résistance complète et le cycle infectieux est ou non interrompu dès son début.

La recherche d'une résistance générale peut présenter plus de difficultés pour 3 raisons : elle est susceptible d'intervenir à chacune des étapes successives d'un cycle infectieux en réglant la vitesse de déroulement, elle agit sur l'enchaînement des cycles successifs pendant tout le cours de l'épidémie, elle s'évalue quantitativement. Aux symptômes caractéristiques qui permettent d'identifier la maladie s'ajoutent d'autres signes qui traduisent les multiples interactions entre les diverses armes de l'agent pathogène et les différents moyens de défense de l'hôte.

Ainsi, les trachéomycoses sont aisément reconnues par le flétrissement de la plante malade. C'est le cas de la verticilliose de la tomate provoquée par $V$. dahliae. Mais la résistance générale de la tomate à cette maladie se manifeste aussi par la durée de la période d'incubation, l'étendue et l'intensité des altérations foliaires et l'importance des troubles de la croissance et du développement.

L'étude des critères de sélection de la résistance générale pour ce pathosystème est justifiée par le fait qu'il n'y a pas d'évidence indiscutable de l'existence d'une relation gène pour gène au sens de FLOR (1956). OKIE \& GADNER (1982) ont montré que le gène $V e$ ne confère qu'une tolérance au Verticillium, au moins pour certains cultivars de tomate qui le possèdent, et, selon PEGG (1974), la résistance à la race 2 est partielle, dépendante du milieu et vraisemblablement polygénique.

Dès lors, le manque d'unanimité dans le choix d'un effet unique de la verticilliose pour évaluer le niveau de résistance générale des variétés de tomate n'est pas surprenant. LOODBROOK (1933) a proposé la durée de la période d'incubation. Pour VISSER \& HATTING (1980), c'est l'intensité des altérations foliaires qui constitue une réponse quantitative de l'hôte à l'infection par $V$. dahliae et permet des comparaisons entre variétés: WALKER et al. (1954), SINHA \& WOOD (1967), BUCHENAUER \& ERWIN (1976) prennent en compte l'étendue des altérations foliaires. BEN YePheT \& PILOWSKY (1979), PEGG (1965) utilisent la taille de la plante pour évaluer la sensibilité de la tomate ; quant à ALEKSIC et al. (1972), ils mesurent la sensibilité du poivron à la verticilliose par un indice de rabougrissement.

Ce choix d'une manifestation unique de la maladie pour évaluer le niveau de résistance générale d'une variété ou comparer plusieurs variétés est une simplification pratique. Cette simplification est justifiée si les diverses réponses élémentaires de la plante à la maladie sont constamment liées et sont sujettes à des variations toujours de même sens et de même intensité. Dans le cas contraire, ce choix présente un risque. Des éléments du patrimoine génétique qui contribuent à protéger l'espèce mais ne gouvernent pas la manifestation prise en compte peuvent être perdus au cours des programmes de sélection, exactement comme peut être perdu un bon niveau initial de résistance générale par la seule prise en considération de la résistance spécifique; on ne peut exclure qu'un tel choix génère l'homologue de l'effet "vertifolia » décrit par VAN DER PLANK (1968).

Pour tenter de répondre à ces interrogations, nous avons comparé les divers effets d'un même clone de $V$. dahliae sur la croissance et le développement de 5 variétés de tomate.

\section{MATÉRIEL ET MÉTHODES}

\section{A. Agent pathogène}

L'isolat initial de Verticillium dahliae a été obtenu, en 1978, à partir d'une tige de tomate atteinte de verticilliose dans la région de Casablanca (Maroc).

SEBTI (1982) en a isolé plusieurs clones par repiquage de microconidies. L'un de ces clones (C19) est caractérisé par un mycélium blanc cotonneux, une sclérogenèse précoce et bien visible, une stabilité et une croissance satisfaisante. Conservé sous azote liquide, il est repris et utilisé pour toutes les inoculations. Il est cultivé à $26^{\circ} \mathrm{C}$ à l'obscurité sur milieu pomme de terre glucose gélosé additionné d'extrait de levure à $1 \mathrm{~g} / 1$ (PDA-YE). Les conidies sont mises en suspension dans de l'eau distillée stérile par grattage de cultures âgées de $10 \mathrm{j}$.

\section{B. Hôte}

Cinq variétés de tomate ont été comparées : " Roma VF », " Marmande VR », " Raf », "Supermarmande », « Red jacket ». Les 2 premières portent le gène $V e$ de résistance spécifique à la race commune de $V$. dahliae. Les semences ont été aimablement fournies par les Ets ClausE.

\section{Dispositif expérimental}

Toute l'expérience a été réalisée simultanément dans une même serre, sous une photopériode de $12 \mathrm{~h}$, à température voisine de $26^{\circ} \mathrm{C}$ le jour et de $18{ }^{\circ} \mathrm{C}$ la nuit et avec une humidité relative de l'air stabilisée à environ 60 p. 100 de la saturation.

Les semences sont enfouies à $1 \mathrm{~cm}$ de profondeur dans des pots en plastique contenant $400 \mathrm{ml}$ de tourbe de Hollande minéralisée (TKS2, Floratorf). Une solution nutritive à $1 \mathrm{ml} / 1$ (LIFAN, BAYER) est apportée une fois, 7 semaines après le semis pour compenser l'épuisement de la tourbe. Huit lots de 11 plantes de vigueur comparable sont constitués pour chaque variété et sont répartis selon un dispositif en bloc aléatoire complet avec 4 répétitions : à chaque bloc sont affectés, de façon aléatoire, 2 lots de chacune des variétés, l'un destiné à être contaminé, l'autre servant de témoin. L'analyse statistique des résultats a été effectuée au moyen du logiciel SPSS (a Statistical Package for the Social Sciences) implanté à l'Univac 1110 du Centre Paris-Sud Informatique. 


\section{Inoculation}

En 1982, SEBTI a montré que des plants de tomate contaminés au stade 5 feuilles présentent une période d'incubation assez courte et une montée rapide des symptômes : ces derniers évoluent beaucoup moins vite quand l'inoculation est faite au stade une feuille. C'est pourquoi nous avons contaminé le plant en place dans les pots au stade intermédiaire de 3 feuilles, afin de provoquer une maladie qui évolue à un rythme commode à observer.

La méthode d'inoculation associe une bonne probabilité de contamination à un minimum de traumatisme pour la plante ; ceci pour ne pas amplifier ni sousestimer les réponses mesurées.

Le collet des plantes est dégagé de la tourbe pour faire apparaître des radicelles puis l'ensemble est circonscrit à l'aide d'une tige métallique de $6 \mathrm{~mm}$ de diamètre, ce qui blesse légèrement les radicelles supérieures. Les plantes contaminées reçoivent à la base $1 \mathrm{ml}$ d'une suspension de conidies ajustée à $10^{7}$ spores $/ \mathrm{ml}$; les témoins, un volume égal d'eau distillée stérile.

\section{E. Notations}

\section{Durée de la période d'incubation}

Les plantes sont observées chaque jour et l'on note $l^{\prime}$ 'apparition du $1^{\text {er }}$ signe foliaire de verticilliose : épinastie, chlorose ou flétrissement suivant les cas. Les plantes qui n'ont manifesté aucun symptôme au bout d'un mois sont considérées comme saines.

\section{Progression des symptômes foliaires}

La progression des différents symptômes foliaires décrits ci-dessous de la base vers le sommet de la tige est observée tous les $7 \mathrm{j}$ pendant 6 semaines. Une note est attribuée à chaque plante, en fonction de la hauteur atteinte, selon le barème :

0 : plante sans symptôme foliaire

1 : un des symptômes foliaires entre le collet et le $1 / 5$ de la hauteur de la plante

2 : symptômes jusqu'aux $2 / 5$ de la hauteur

\begin{tabular}{|c|c|c|c|c|c|}
\hline & 》) & 》) & 3 & 》 & 》) \\
\hline 4: & $\gg$ & $\gg$ & $4 / 5$ & 》) & $»$ \\
\hline
\end{tabular}

5: " sur toute la hauteur ou plante morte.

Un indice d'étendue des symptômes foliaires (I.E.S.F.) est ensuite calculé pour chaque lot de plantes selon la formule :

$$
\text { I.E.S.F. }=\frac{\text { somme des notes }}{\text { maximum possible }} \times 100
$$

dans laquelle le maximum possible est égal à 5 fois le nombre des plantes.

\section{Indice d'altération foliaire (I.A.F.)}

Calculé chaque semaine pendant 6 semaines, il exprime l'intensité des altérations foliaires. A chaque relevé, une note est attribuée à chaque feuille :

0 : feuille d'aspect sain

1 : épinastie ou feuille flétrie sans chlorose

2 : une ou plusieurs plages légèrement chlorotiques sur 1 ou plusieurs folioles

3 : plages chlorotiques sur toute la surface d' 1 ou plusieurs folioles ou plage chlorotique à centre nécrosé sur 1 ou plusieurs folioles

4 : nécrose complète de plusieurs folioles ou feuille morte.

Un indice d'altération foliaire est ensuite établi pour chaque plante.

$$
\text { I.A.F. }=\frac{\text { somme des notes }}{\text { maximum possible }} \times 100 .
$$

Le maximum possible est égal à 4 fois le nombre de feuilles bien développées portées par la plante.

\section{Croissance}

La partie épicotylée de la plante est mesurée chaque semaine pendant 6 semaines.

\section{Emission des feuilles}

Les feuilles bien développées sont dénombrées une fois par semaine pendant 6 semaines.

\section{Floraison}

L'ensemble des fleurs formées sur les 3 premières inflorescences a été dénombré sur chaque plante à la fin de l'expérience.

\section{RÉSULTATS}

\section{A. Durée de la période d'incubation}

Toutes les variétés sont sensibles au clone $C 19$ de $V$. dahliae. Le gène $V e$ n'interdit pas la manifestation de la maladie sur les variétés qui le possèdent « Roma VF » et "Marmande VR ». Chez ces 2 variétés, la durée de la période d'incubation n'est pas statistiquement différente de la durée moyenne observée chez " Red jacket » et " Raf ». En revanche, "Supermarmande " se distingue des autres à l'exception de "Raf » par une apparition plus précoce des $1^{\text {ers }}$ signes de verticilliose (tabl. 1).

TABLEAU 1

Durée de la période d'incubation, en jours. Incubation period (days).

\begin{tabular}{ccccccc}
\hline \hline Variétés & "Red jacket & " Marmande VR & “Supermarmande " & "Raf » & 《Roma VF " \\
\hline Durée en jours & $15,40 \pm 1,10$ & $15,40 \pm 0,70$ & $13,45 \pm 0,80$ & $14,51 \pm 0,78$ & $15,50 \pm 1,20$ \\
\hline \hline
\end{tabular}




\section{B. Progression des symptômes foliaires}

La progression de ces dommages en direction de l'apex de la plante pendant tout le cours de l'expérience (fig. 1) fait apparaître 3 comportements différents :

La variété " Roma VF », lors de la $1^{\text {re }}$ observation, présente des altérations foliaires sur la portion inférieure de l'axe aérien de même étendue relative que chez "Marmande VR » et " Red jacket ", mais ces altérations ne progressent pas au-delà. La poursuite de l'élongation de la tige entraîne même une réduction de l'indice d'étendue des symptômes foliaires.

Les altérations foliaires s'étendent rapidement et pendant toute la durée des observations chez " Supermarmande " et " Raf ».

Chez « Marmande VR » et « Red jacket », la progression des dommages atteint un palier nettement avant la fin des observations. Par l'étendue des symptômes foliaires, ces 2 variétés se classent entre les plus affectées, "Supermarmande » et "Raf », et la moins atteinte, « Roma VF ».

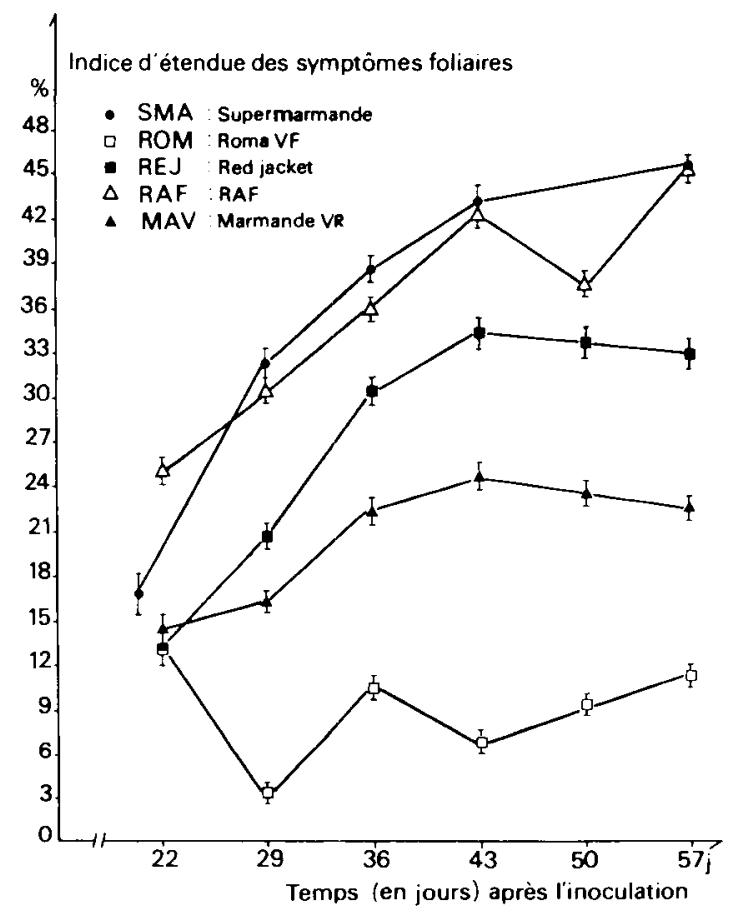

Figure 1

Etendue des symptômes foliaires ;

Intervalle de confiance au seuil de 95 p. 100

Extent of foliar symptoms ; $95 \%$ confidence interval.

\section{Intensité des altérations foliaires}

L'évolution de l'I.A.F. (fig. 2), permet de séparer les 3 mêmes groupes de variétés pendant les 3 premières semaines d'observations. "Roma VF » se singularise par un indice très bas. "Raf " et "Supermarmande ", d'une part, "Red jacket " et " Marmande VR », d'autre part, forment 2 groupes statistiquement différents mais les indices d'un même groupe ne sont pas statistiquement différents. En fin d'expérience, ces groupes se dissocient et chaque variété est caractérisée par un indice propre.

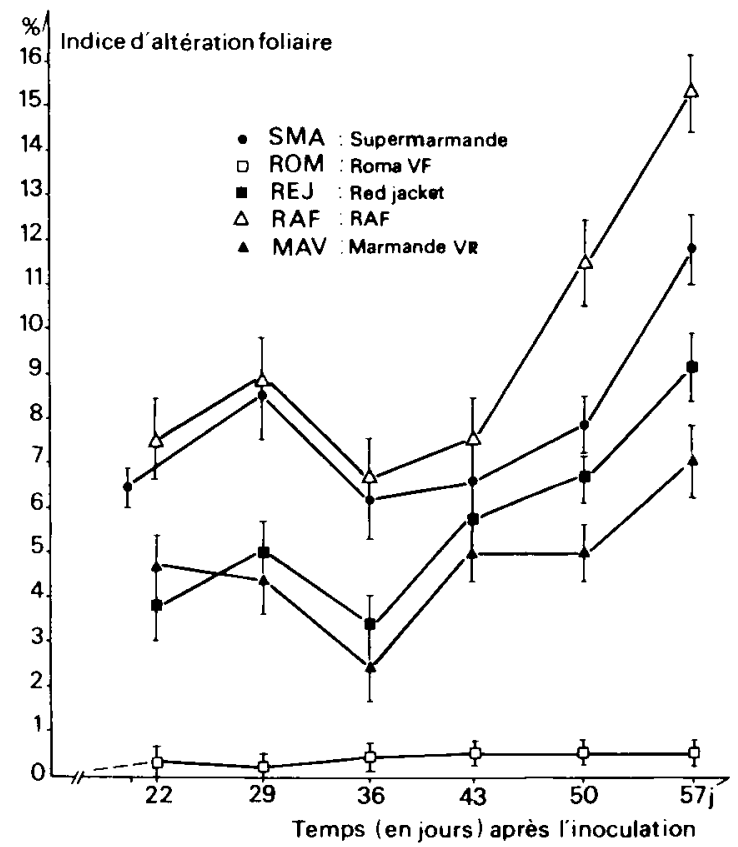

Figure 2

Evolution des indices d'altération foliaire

Intervalle de confiance au seuil 95 p. 100.

Development of the index of leat damage ; $95 \%$ confidence interval.

\section{Croissance de la tige}

Les premiers effets de la verticilliose sur la croissance de l'apex (fig. 3) sont très marqués et de sens différents selon les variétés. "Raf ", seule, réagit à l'inoculation par une élongation accrue. Les 4 autres ont une taille inférieure à celle des témoins non contaminés mais blessés de la même façon. L'action nani-

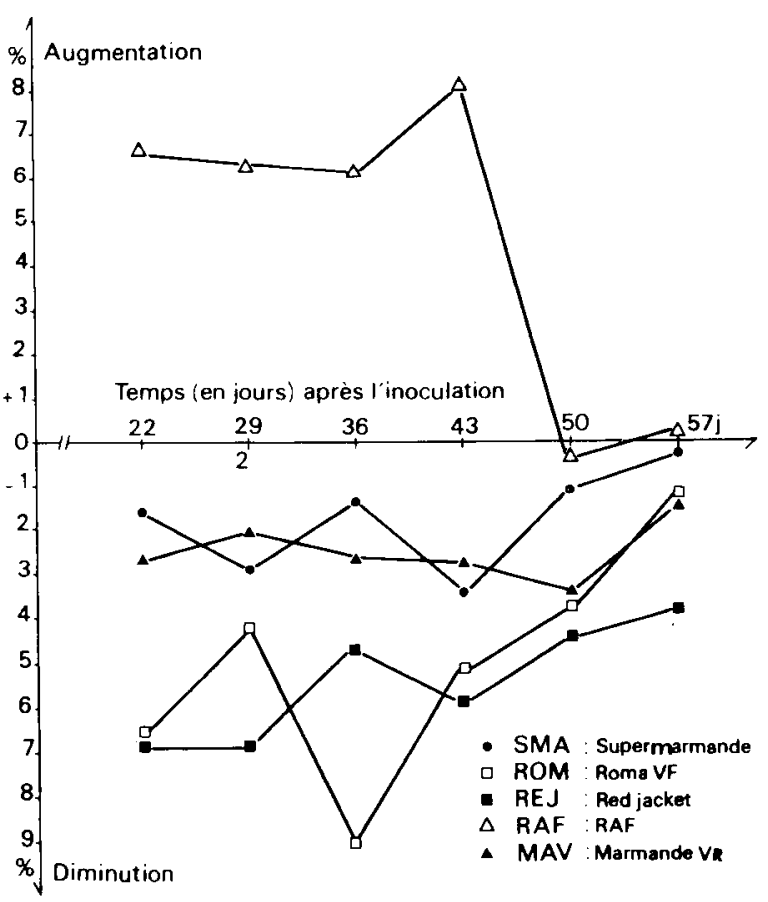

Figure 3

Variation en p. 100 de la hauteur de l'axe aérien des plantes contaminées par rapport aux témoins de la même variété. Variation in the height of the inoculated plants as a percentage of the height of the control. 
sante de $\boldsymbol{V}$. dahliae est légère chez "Supermarmande » et «Marmande VR », plus intense chez « Roma VF » et « Red jacket ».

Ces effets disparaissent ensuite ou s'atténuent fortement chez toutes les variétés à l'exception de « Red jacket " : la diminution de la taille de son axe aérien est encore appréciable $50 \mathrm{j}$ après l'inoculation. En d'autres termes, " Raf » anticipe, "Supermarmande », «Roma VF » et «Marmande VR » diffèrent leur croissance et « Red jacket » la freine durablement.

\section{E. Emission des feuilles}

On observe que la verticilliose stimule, chez la variété «Supermarmande », l'émission des feuilles tout au long de l'expérience (fig. 4). Chez la variété "Roma VF", cette stimulation est encore plus intense mais temporaire : au-delà de 4 semaines après la fin de la période d'incubation, la production de feuilles est au contraire fortement déprimée. Les 3 autres variétés « Marmande VR », « Raf » et « Red jacket » ont un comportement inverse : le ralentissement initial est finalement plus que compensé car, en fin d'expérience, les plantes contaminées comportent plus de feuilles que les témoins.

\section{F. Floraison}

On distingue les effets directs et différentiels de la maladie sur le nombre de fleurs (tabl. 2). La diminution du nombre de fleurs est statistiquement significative pour toutes les variétés, mais certaines sont plus affectées que d'autres. Si l'on rapproche les variétés qui réagissent de façon similaire, on peut distinguer

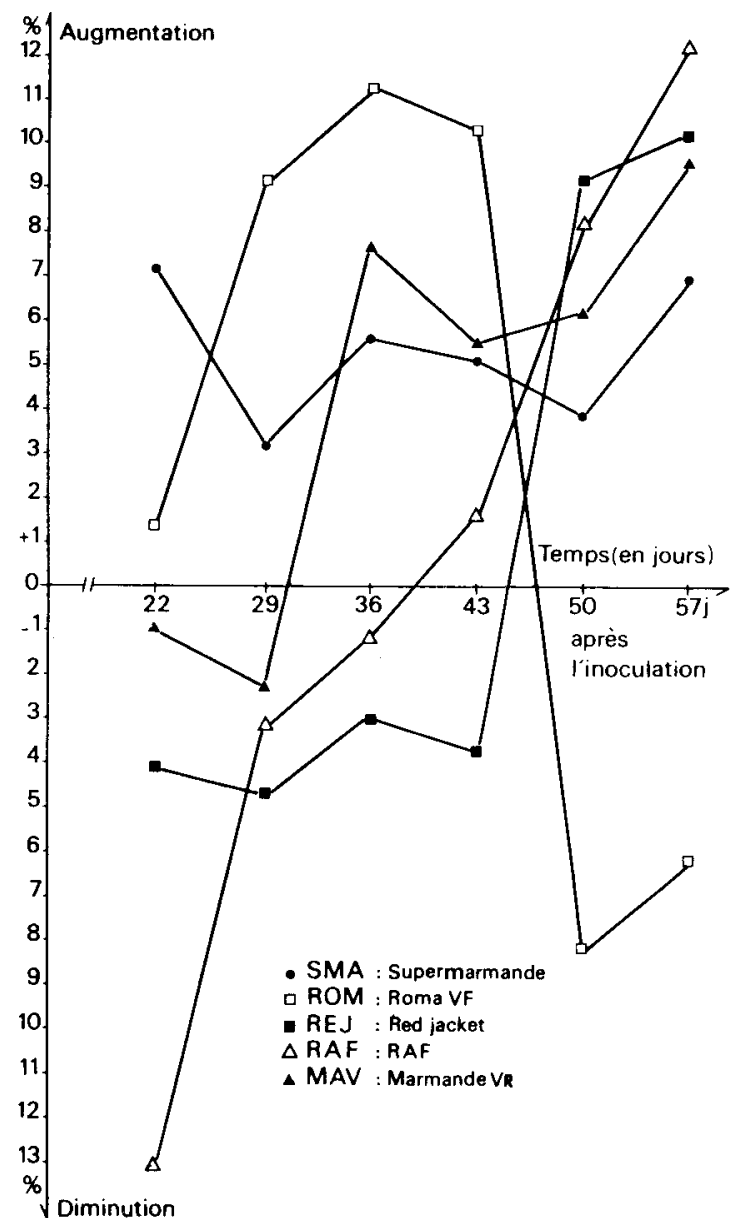

Figure 4

Variation en p. 100 du témoin de nombre de feuilles émises par les plantes contaminées.

Variation in the number of leaves in inoculated plants as a percentage of the number of the leaves in the control.

TABLEAU 2

Effet de la verticilliose sur la floraison : nombre de fleurs sur les 3 premières inflorescences.

Effect of Verticillium wilt on flowering : number of flowers on the first three floriferous nodes.

\begin{tabular}{|c|c|c|c|c|c|c|}
\hline & & \multicolumn{5}{|c|}{ Variétés } \\
\hline & & "Red jacket $»$ & «Marmande VR & Supermarmande » & «Raf » & «Roma VF» \\
\hline \multirow{2}{*}{ Nombre moyen de fleurs } & Témoin & $10,45 \pm 0,55$ & $9,25 \pm 0,53$ & $11,40 \pm 0,90$ & $10,09 \pm 0,50$ & $8,34 \pm 0, \dot{58}$ \\
\hline & Contaminé & $7,20 \pm 0,60$ & $7,00 \pm 0,88$ & $7,77 \pm 0,74$ & $6,43 \pm 0,48$ & $6,20 \pm 0,80$ \\
\hline Différence des moyennes & & $3,25^{\circ}$ & 2,25 & 3,63 & 3,66 & 2,14 \\
\hline \multirow{4}{*}{$\begin{array}{l}\text { Interactions : } \\
\text { Probabilité du } \\
\text { F calculé }\left(^{*}\right)\end{array}$} & «Red jacket » & - & 0,13 & 0,58 & 0,54 & 0,16 \\
\hline & « Marmande VR » & & - & 0,04 & 0,03 & 0,88 \\
\hline & « Supermarmande» & & & - & 0,97 & 0,07 \\
\hline & « Raf » & & & & - & 0,06 \\
\hline
\end{tabular}

$\left({ }^{*}\right)$ Analyse de variance à 2 critères de classification (variété et traitement) pour les variétés prises 2 à 2 . Le rapport $\mathrm{F}=$ carré moyen de l'interaction/carré moyen résiduel. Une probabilité faible de $\mathrm{F}$ est l'indice d'une interaction variété $\times$ traitement, e'est-à-dire d'une différence de réponse entre les 2 variétés. Par exemple, sur ce critère, "Roma VF » se distingue à la fois de "Supermarmande " et de " Raf », et ressemble á « Marmande VR ».

Analysis of variance with two classification criteria (variety and treatment). The $\mathrm{F}$ ratio = interaction mean square/residual mean square. A low $\mathrm{F}$ probability indicates a variety-treatment interaction, i.e. a difference in response between the two varieties. On this criterion, for instance, "Roma VF " differs both from "Supermarmande " and " Raf », and is similar to " Marmande VR ". 
2 types de réponses : " Roma VF " et " Marmande VR » sont relativement moins affectées, mais elles ont aussi un peu moins fleuri que les autres variétés. Les 3 autres variétés sont sensiblement plus atteintes.

\section{DISCUSSION - CONCLUSION}

Cette $1^{\text {re }}$ étude, prenant en compte plusieurs paramètres d'observation symptomatologique, montre à l'évidence qu'un même clone de $\boldsymbol{V}$. dahliae entraîne des réponses différentes de la part des 5 variétés de tomate.

La différence entre variétés peut être qualitative pour un paramètre considéré. Il en est ainsi pour la croissance de l'axe aérien : elle est accélérée chez la variété « Raf », elle est déprimée chez les 4 autres. De même, la verticilliose, dans un premier temps, stimule l'émission des feuilles chez "Roma VF » et la réduit chez « Red jacket » et " Raf ». Enfin, 2 réponses de sens opposé peuvent se succéder dans un ordre inversé : le nombre de feuilles déployées par la variété " Roma VF » est dans un $1^{\text {er }}$ temps accru par la verticilliose et dans un $2^{\mathrm{e}}$ temps diminué ; l'inverse est observé chez les variétés « Red jacket » et « Raf».

Des effets de même sens, mais quantitativement différents selon les variétés, sont plus fréquemment notés. La réduction de la floraison est constante mais les 5 variétés constituent 2 classes statistiquement distinctes. Chez toutes les variétés, les feuilles sont affectées par la verticilliose, mais la progression de l'indice d'altération foliaire, nulle chez "Roma VF » après l'apparition des premiers dommages, est forte chez "Raf " et "Supermarmande ", intermédiaire chez « Red jacket » et "Marmande VR » : l'indice final sépare nettement les 5 variétés. Des observations analogues sont faites à propos de l'évolution des indices d'étendue des symptômes foliaires.

Certains effets de la verticilliose varient simultanément et dans le même sens chez les 5 variétés. Ainsi, toutes se classent dans le même ordre pour l'étendue et l'intensité des dommages foliaires. L'inoculation d'autres clones de $\boldsymbol{V}$. dahliae à ces mêmes variétés ou l'inoculation du même clone $C 19$ à d'autres variétés pourrait permettre de dissocier ces 2 effets de la maladie. Cependant le choix des symptômes observés étant arbitraire, il se peut aussi qu'ils relèvent des mêmes mécanismes physiologiques et que ceux-ci mettent en jeu la même part du génome de la tomate.

Mais la plupart des réponses de l'hôte à l'infection sont apparemment sans lien constant entre elles.
Quand les variétés « Raf » et " Red jacket » traduisent toutes deux la maladie par un ralentissement du déploiement de leurs feuilles, la $1^{\text {re }}$ accélère la croissance de sa tige et la $2^{e}$ la freine. Les feuilles de la variété " Roma VF » sont très peu altérées et les dommages foliaires initiaux ne s'étendent pas d'une manière appréciable; en revanche, les perturbations de la croissance de l'axe aérien et du développement des feuilles sont profondes et durables. Cette absence de liaison entre des expressions différentes de la maladie peut signifier que, chez l'hôte, des gènes ou des systèmes géniques différents y participent.

Ces $1^{\text {res }}$ observations apportent des indications pour la conduite des programmes d'amélioration de la résistance générale de la tomate à $V$. dahliae.

Il apparaît clairement que l'évaluation générale du niveau de cette résistance ne peut être rationnellement fondée sur l'estimation d'un seul effet de la maladie : aucun n'en rend complètement compte parce qu'aucun n'est l'expression de l'ensemble des gènes qui gouvernent globalement la résistance générale.

D'autre part, si l'on classe les 5 variétés, aucune ne présente le comportement optimum vis-à-vis de l'ensemble des effets de la verticilliose. La variété " Roma VF » est la mieux protégée contre les altérations foliaires mais son développement, à en juger par l'émission des feuilles, est profondément perturbé. La variété "Supermarmande", la moins sensible aux effets de $V$. dahliae sur la croissance de la tige et la formation de l'appareil foliaire se classe au rang des plus vulnérables aux actions du champignon sur le fonctionnement de ses feuilles. La variété «Marmande VR » figure parmi celles dont la floraison est la moins déprimée par la maladie mais sa croissance est fortement retardée. Un programme qui tient compte de ces différentes interactions entre l'hôte et l'agent pathogène peut conduire à une variété réunissant les caractéristiques avantageuses actuellement dispersées et présenter, de ce fait, une résistance générale plus efficace.

Reçu le 28 mai 1984. Accepté le 16 novembre 1984.

\section{REMERCIEMENTS}

Les auteurs remercient M. le Professeur Chevaugeon pour ses nombreux avis et conseils dans la préparation et la réalisation du texte. Ils remercient également $M$. TAILLER pour son aide technique ainsi que $M$. Guenaro des Ets Clause pour la fourniture des semences.

\section{RÉFÉRENCES BIBLIOGRAPHIQUES}

\begin{abstract}
Aleksic Z., Aleksic D., Sutic D., 1972. La pénétration du Verticillium albo-atrum Reinke \& Bert. dans le poivron (Capsicum spp.) différemment résistant au parasite. Actas III Congrès Un. fitopatol. mediterr., Oeiras, 22-28 Outubro, 535-539.
\end{abstract}

Ben Yephet Y., Pilowsky M., 1979. A method for determining susceptibility of tomatoes to Verticillium dahliae in the greenhouse. Plant Dis. Rep., 63, 66-69.
Buchenauer H., Erwin D. C., 1976. Effect of plant growth retardant pydanon on Verticillium wilt of cotton and tomato. Phytopathology, 66, 1140-1143.

Clerjeau M., Laterrot H., Pitrat M., 1979. Création de variétés résistantes aux maladies chez les plantes maraîchères. Bull. Tech. Inform. Min. Agric., 337, 101-114.

Flor H. H., 1956. The complementary genic systems in flax and flax rust. $A d v$. Genet., 8, 29-54. 
Loodbrook W. V., 1933. Pathogenicity and environmental studies on Verticillium hadromycosis. Phytopathology, 23, 117-154.

Okie W. R., Gadner R. G., 1982. Screening tomato seedlings for resistance to Verticillium dahliae races 1 and 2. Plant Dis., 66, 3437.

Pegg G. F., 1965. Phytotoxin production by Verticillium alboatrum Reinke and Berthold. Nature, 208, 1228-1229.

Pegg G. F., 1974. Verticillium disease. Rev. Plant Pathol., 53, $157-$ 182.

Sebti S., 1982. Essai d'analyse des composantes du pouvoir pathogène de Verticillium albo-atrum Reinke et Bert. Thèse Doct. $3^{\text {e }}$ Cycle. Univ. Paris XI, Orsay.
Sinha A. K., Wood R. K. S., 1967. An analysis of responses of resistant and susceptible tomato plants to Verticillium infection. Ann. Appl. Biol., 59, 143-154.

Van Der Plank J. E., 1968. Disease resistance in plants. Acad. Press Inc. New York, London, 204 p.

Visser S., Hatting J., 1980. Criteria for quantal host response of tomato plants to infection by Verticillium dahliae. Plant Dis., 64, 207-208.

Walker J. C., Gallegly M. E., Bloom J. R., Scheffer R. P., 1954. Relation of plant nutrition to disease development VIII : Verticillium wilt of tomato. Am. J. Bot., 41, 760-762. 